\title{
Role of Utility and Inference in the Evolution of Functional Information
}

\author{
Alexei A. Sharov \\ Received: 14 March 2008 / Accepted: 16 May 2008 / \\ Published online: 4 March 2009 \\ (C) National Institute on Aging 2009
}

\begin{abstract}
Functional information means an encoded network of functions in living organisms from molecular signaling pathways to an organism's behavior. It is represented by two components: code and an interpretation system, which together form a self-sustaining semantic closure. Semantic closure allows some freedom between components because small variations of the code are still interpretable. The interpretation system consists of inference rules that control the correspondence between the code and the function (phenotype) and determines the shape of the fitness landscape. The utility factor operates at multiple time scales: short-term selection drives evolution towards higher survival and reproduction rate within a given fitness landscape, and long-term selection favors those fitness landscapes that support adaptability and lead to evolutionary expansion of certain lineages. Inference rules make short-term selection possible by shaping the fitness landscape and defining possible directions of evolution, but they are under control of the long-term selection of lineages. Communication normally occurs within a set of agents with compatible interpretation systems, which I call communication system. Functional information cannot be directly transferred between communication systems with incompatible inference rules. Each biological species is a genetic communication system that carries unique functional information together with inference rules that determine evolutionary directions and constraints. This view of the relation between utility and inference can resolve the conflict between realism/positivism and pragmatism. Realism overemphasizes the role of inference in evolution of human knowledge because it assumes that logic is embedded in reality. Pragmatism substitutes usefulness for truth and therefore ignores the advantage of inference. The proposed concept of evolutionary pragmatism rejects the idea that logic is embedded in reality; instead, inference rules are constructed within each communication system to represent reality, and they evolve towards higher adaptability on a long time scale.
\end{abstract}

\footnotetext{
A. A. Sharov $(\bowtie)$

Laboratory of Genetics, National Institute on Aging, 251 Bayview Boulevard,

Baltimore, MD 21224, USA

e-mail: sharoval@mail.nih.gov
} 
Keywords Biosemiotics · Pragmatism - Umwelt - Semantic closure ·

Self-organization $\cdot$ Inference $\cdot$ Evolution

\section{Introduction: Functional Information, Knowledge, and Umwelt}

Information theory explores the statistical properties of signals but entirely ignores the meaning of the transmitted messages. Biosemiotics attempts to fill this gap by studying the meaning of the various kinds of information that are used by living organisms, including DNA sequences, cell signaling, pheromones, acoustic communication, and others (Sharov 1992; Hoffmeyer 1996). The main feature of information, in living systems, is that it is functional, which means that it is necessary for the performing of some function at the level of a cell, organ, or whole organism. Some of these functions are needed continuously (e.g., metabolism) others are induced at specific stages of development or in relatively rare situations (e.g., danger). Genetic information is often interpreted as a sequence of commands to perform specific actions that result in growth, development, survival, and reproduction. A better metaphor would be a book of recipes, or know-how, that is activated when an organism needs a specific function. Functional information can be viewed as a simplified form of knowledge. In other words, I assume that knowledge is a natural phenomenon which originated long before humans.

This approach was first proposed by von Uexküll (1982), who noticed that the activities of each living organism are based on an internal model of the world that he called Umwelt. This model includes memory of external objects, relations between objects, and recipes for their utilization. Animals know their shelters, nests, food sources, hunting strategies etc., and all these help them to survive and reproduce. Umwelt makes external objects meaningful for the animal; thus, Uexküll viewed his work as a theory of meaning. Uexküll went further and assumed that Umwelt exists even in organisms without a brain, such as plants and single cells, because they also behave as if they had a model of their environment. At that time it was not clear where this model was memorized; however, now we know more about cellular information processing. The DNA sequence, in the nucleus of each cell, contains an enormous amount of information which can be viewed as a long-term memory, passed through generations with only minor changes per generation. In addition, chromatin structure in the nucleus can carry short-term individual memory that can be updated on demand. Thus, a nucleus functions as a storage of functional information within a cell and in this respect it is similar to the brain of animals.

The proposed term "functional information" is similar to Umwelt, but it has several advantages: (1) its meaning is not limited to a model of the environment (Umwelt means "environment" in German) and includes models of the internal structure and function of an organism, (2) it includes both inherited and acquired information, and (3) it emphasizes the pragmatic aspect of information in living organisms. The comparative study of functional information across various taxa is challenging, because humans, animals, plants, and unicellular organisms differ substantially in the way they acquire, process, and store information. I will reserve the term "knowledge" for only those cases of highly organized functional 
information that include a rich network of inferences (e.g., deduction, prediction, and extrapolation). Functional information is heterogeneous even within the same species. In addition to the knowledge stored in the brain, humans have somatic information stored in the nuclei of the other cells of the body. It is debatable whether somatic functional information can be called "knowledge," because we still do not know most of the inference network within the cell. At this point is it better to refer to it as functional information, or experience.

In this paper I discuss two main factors, utility and inference, that guide the emergence and evolution of functional information. By inference I mean rules of interpretation of encoded functional information executed by a complex regulatory network similar to a computer. The inference system controls the correspondence between the genome sequence and the phenotype, and determines the shape of the fitness landscape. The utility factor operates at multiple time scales: short-term selection drives evolution towards higher survival and reproduction rates within a given fitness landscape, and long-term selection favors those fitness landscapes that support adaptability and lead to evolutionary expansion of certain lineages. Inference rules make short-term selection possible by shaping the fitness landscape which defines possible directions of evolution, but it is under control of the long-term selection of lineages.

This view of the relationship between utility and inference can resolve the conflict between realism/positivism and pragmatism. Realism overemphasizes the role of inference in human knowledge because it assumes that logic is embedded in reality, and we can discover the truth by following logic irrespective of utility. In contrast, pragmatism substitutes usefulness for truth and therefore ignores the advantage of inference. The proposed concept of evolutionary pragmatism rejects the idea that logic is embedded in reality; instead, inference systems are constructed to represent reality. In the short term, living systems search for maximum utility within a given inference system, but inference systems themselves evolve over a long time scale according to their adaptability.

\section{Biological Functions have a Semiotic Nature}

The notion of function is not present in the vocabulary of physics. Physics studies force and work, but this is abstract force and work, with no function (Kauffman 2000). We understand function as activity that achieves the goal of an agent or increases its value; in other words, function is inherently a teleological notion. In biology, function is often opposed to structure, as if structure is not functional. However, the body is a result of early morphogenetic functions, and thus it is functional too. Hence, an organism's morphology encoded in the genome is a part of its functional information. Goal-directed activity differs from physical causation, because the goal is actively chosen by an agent rather than being set in a deterministic or stochastic way. This choice is stored in the form of a code (e.g., DNA sequence or memory) and can be addressed/activated on demand. Choice is based on preferential storage of a code, and functional behavior has to be encoded in order to be chosen. Therefore, functional behavior always has a semiotic nature (Pattee 1982; Rosen 1985) and is represented by a code. 
The second important component of functional information is an interpretation mechanism that converts the code into various actions/functions, ranging from catalysis and metabolism to morphogenesis and behavior. The network of actions can be described by Petri nets (Sharov 1991) or production relations (Ackoff and Emery 1972). Interestingly, the network of actions supports both the code and the interpretation system, which makes a self-sustaining and self-replicating functional loop called a semantic closure (Pattee 1982). Understanding of the semantic closure as a self-sustaining loop delineates the boundary between biosemiotics and traditional science, which is focused on studying only linear causality. Information theory disjoints the semantic closure by assuming that interpretation is fixed for all possible codes.

An example of the information-theory approach is a model of natural selection which is based on a direct correspondence between genotypes and phenotypes, assuming that interpretation of genetic information is fixed. Each phenotype is then characterized by fitness measured by a Malthusian parameter that integrates survival and reproduction (Fisher 1930). In combination, these two assumptions lead to the concept of fitness landscape, where fitness is assigned to each genotype. This model has a certain merit because it demonstrates evolutionary change towards higher fitness, (i.e., utility), but it fails to explain the evolution of interpretation mechanisms because interpretation is fixed by definition. This model creates the illusion that immediate utility is the only factor that drives biological evolution, an idea blindly accepted by the majority of biologists. My goal is to show that the interpretation mechanism, and its associated inference system, is as important in evolution as utility and should not be ignored, even if we do not have well-developed mathematical models to describe it.

Code and interpretation systems do not exist without each other because the interpretation system is renewed and repaired by reading and interpreting the code, and the code cannot be read and interpreted without the interpretation system. Different portions of the code store the information needed to interpret each other and to modify this interpretation depending on the environment; thus, together, they determine the adjustable structure of the interpretation network. If we received a genome sequence of an extraterrestrial organism from another galaxy via radio transmission, we would never be able to decipher it. The only way to understand the genome is to observe how living cells read and interpret it. Thus, the code by itself has no meaning. It may have regularities and autocorrelations that we can interpret in our own way, but it is impossible to guess what these regularities were originally designed for. However, the code and the interpretation systems are partially independent because the same interpretation system may work to interpret reasonable modifications of the code, and the same code can be interpreted by slightly different interpretation systems. Thus, there is room for some wiggling within the semantic closure. Because the interpretation system is self-renewed by reading the code, it will eventually change to a new state after interpreting the code. This new steady state is generally not uniquely defined by the code and depends on the initial state of the interpretation system. For example, cloned animals have the same DNA sequence as their nuclear parent, but their phenotype is different because of malfunctions in the interpretation system. 
The interpretation system is dynamic and depends on the environment. This dependency is largely encoded in a set of sensors and signal transduction networks which control the homeostasis of an organism and increase chances of survival. However, these transient changes do not affect the fitness landscape, because fitness is calculated from the cumulative survival and reproduction rate through an entire life cycle. In contrast, long-term irreversible changes in the environment (e.g., through climate change or dispersal of organisms to another climate zone) may cause permanent changes within the interpretation system. The initial phase of adaptation may involve changes in habit selection, when organisms attempt to find conditions that match their old environment, but eventually these changes affect genetic selection and result in the reshaping of the fitness landscape. Thus, interpretation of the code is neither unique nor automatic, but depends on the interpretation system, which in turn depends on the environment.

For the sake of argument, let us assume that the state of the interpretation system is indeed uniquely determined by the code and all alternative states are unstable and/ or not viable. It appears that even in this oversimplified case the role of the interpretation system is nontrivial and we still need the notion of semantic closure. The reason is that the phenotype is not computable for all modifications of the genome sequence, i.e., the genome is too complex for global predictions. The only way to handle calculations is to make them local by limiting genomic changes to a small section of DNA (e.g., within one gene or promoter of a gene). If this section of DNA contributes to the survival and reproduction rate of the organisms, then it is possible to predict the fitness landscape and the direction of evolutionary change towards higher fitness. However, we should not expect the fitness landscape to remain constant, because the interpretation system changes and so does the correspondence between the sequence of our selected DNA segment and the phenotype. The interpretation system depends on the entire genome as well as on the environment and is not fully computable; therefore, it is impossible to predict the global fitness landscape for the whole genome. Even in a chess game with only 32 figures, 64 positions and no environmental factors, we cannot predict fitness of each board position for each figure. An organism "game" has at least 25,000 types of figures (i.e., genes) and the number of figures (molecules) is estimated at millions per cell. Thus it is not surprising that evolution is not fully predictable, and this is not merely a limitation of human knowledge about life but a feature of life itself (Rosen 1991).

Consider the evolution of insect wings as an example of cross-interpretation of system components, where fitness landscape for one gene is affected by the evolution of other genes. Wings did not appear as an adaptation for flying because there was no flying function yet. Wing-resembling appendages may have helped insects to glide while falling down from trees. As a result, a fitness gradient appeared for genes that can modify the size and shape of these appendages, i.e., the fitness landscape was modified by evolving behavior. As gliding progressed, insects changed their behavior and started bending their pre-wings for steering in the air. This created a fitness gradient for genes affecting corresponding muscles and cuticle thickness at the base of pre-wings. Again, the interpretation system and the fitness landscape changed. Finally, the muscles became stronger and could partially support 
forced flight. As the function changes, so does the fitness landscape for all genes affecting the performance of wings.

Thus, although evolution follows fitness gradients according to Darwin, it eventually leads to a change of the interpretation system which reshapes fitness landscapes for many other genes. Thus, evolution cannot be reduced to genetic selection that follows immediate utility; in addition there is emergence of novel functional interpretations of already existing DNA code. Kauffman (1993) called this emergent property of biological evolution self-organization, but erroneously considered it similar to self-ordering events in the physical world (e.g., dissipative systems) (Abel and Trevors 2006). However, there is little similarity between these phenomena because self-ordering events are not encoded, whereas biological selforganization is a change in the interpretation system which is encoded and inherited. Below I will show that changes in the interpretation system follow specific rules similar to human inference or logic that were selected in macroevolution during long competition between lineages.

\section{Genetic Selection and Habit Selection Increase the Utility of Functional Information}

Utility is an important criterion of functionality. For example, if alteration of a DNA segment negatively affects survival and reproduction of transgenic organisms, then we can conclude that this portion of DNA is a functional code. Utility of genetic information is sustained and increased via natural selection. However, the term "natural selection" suggested by Darwin should not be interpreted as if nature performs the selection of organisms; nature is not an agent and cannot select anything. It is more accurate to call this process "genetic selection," because selected features are encoded genetically (i.e., in chromosomal DNA). Genetic selection is mediated by the ability of organisms to reproduce; thus the act of selection is done by an organism that carries functional information (i.e., it is a self-selection).

Another common mistake is to consider reproduction as a passive copying process (Dawkins 1989). The first objection to this view is that self-reproduction is not passive because it requires energy and know-how for constructing another body. Thus, organisms have to struggle to get this energy as food, and use their functional information (code plus interpretation system). DNA replication differs from crystal growth because nucleic acids are sophisticated molecular instruments optimized for storing and replicating functional information in living organisms. They emerged as a product of a long evolution and were designed for their function. DNA replication is active because it requires special catalyst complexes to do it. Finally, DNA replication is accompanied by complex mechanisms of proofreading that can identify errors and fix them. In comparison, no proofreading occurs in crystals.

Utility of living systems can be sustained and increased by other forms of selection besides genetic. Habit selection is memorizing and repeating specific behavioral patterns if they were useful in an animal's previous experience. In unicellular organisms and plants, habits are most likely encoded epigenetically in the chromatin structure within individual cells. In animals they are encoded in the brain. Mechanisms of memory storage in the brain are not fully understood. But because 
brain memory originated from cellular memory encoded epigenetically, we can speculate that brain memory too may be epigenetic in nature, at least partially. For example, the chromatin structure of neurons may control the pattern of synapse activation. But other mechanisms are also possible. Most habit information is lost with the death of an animal; however, a small portion of it can be transferred to the next generation via teaching and/or copying as well as by epigenetic inheritance.

One of the main problems in biology is whether utility is the only measure of functionality; in other words, can functionality be reduced to utility? Answering "yes" to this question is tempting, because it would greatly simplify the evolutionary doctrine. Then only those features of organisms are important that affect fitness (survival and reproduction rate); all other features have no biological meaning and are not important, either for the organism or for us to study. From this point of view, genetic selection plus habit selection explain all important features of biological evolution.

However, several lines of evidence indicate that utility is not the only factor (and may not be the main one) that controls the evolution of biological functions. Only a small portion of the genome (5\%) is conserved between mammalian species, which indicates a lack of strong genetic selection for most of the genome. Even conserved regions often have no apparent phenotype and no effect on survival and reproduction, based on experiments with mutants and transgenic organisms (Bouché and Bouchez 2001; Ahituv et al. 2007). Interestingly, many genes that have no apparent effect on organism morphology and survival do have molecular functions. Some control proofreading of DNA replication; others enforce nonsense-mediated degradation of mRNA, and so on. We call these processes "functions" because they make sense to us as housekeeping mechanisms of cell homeostasis no matter whether they affect survival and reproduction in normal conditions or not. It may be argued that these processes are functional because they may affect survival and reproduction in some extreme condition, e.g., intensive radiation or heat. But this argument may be a false lead because we have no proof that these functions indeed developed as a response to extreme conditions. It seems equally likely that they developed to cope with internal problems like constant evolutionary change of the interpretation system, which is discussed in more detail below.

\section{Interpretation System Controls Adaptability}

The interpretation system, which is a network of pathways that converts encoded functional information into phenotype and behavior, is a complex hierarchical system. At its lowest level there are intracellular molecular processes, including all steps in gene function (i.e., transcription, initiation and elongation, mRNA splicing and transport, protein synthesis, transport, modification, and assembly into protein complexes), pathways of signal transduction, and metabolism. The next level includes processes that involve assembly and modification of various cellular structures (i.e., chromosome condensation, pinocytosis, membrane assembly and disassembly, ribosome assembly, cytoskeleton assembly and disassembly, secretion, establishment of cellular junctions, assembly of extracellular matrix, etc.). The third level includes tissue growth, differentiation, cell migration, and assembly of large 
extracellular structures (i.e., bones). The fourth level includes coordinated growth and development of organs controlled by various signals, ranging from growth factors and hormones to neural-dependent morphogenesis. At the top level is the neural system, which controls behavior and somatic functions. Change in DNA sequence may cause ripple effects through all levels of the interpretation system but may also be blocked at any level. The interpretation system has enormous homeostatic capacity; it can neutralize many deleterious effects no matter whether they come from the environment or from accidental mutations. Because of this, many mutations appear neutral, with no effect on fitness.

The interpretation system is normally more stable than the code; otherwise it would be meaningless to talk about the code. Thus, if we select a specific portion of a biological code (e.g., gene or mental state), the interpretation system for this portion of the code should change more slowly than the code itself. For example, the quickly changing mental code is interpreted by stable body structures which in turn are products of gene interpretation. Evolution of genes is much slower than changes in animal behavior. Thus, the behavioral status of an animal cannot be viewed as a part of the interpretation system for its genes. However, stable behavioral patterns are encoded genetically and can be viewed as components of the interpretation system for other genes. In the previous example of flight origin in insects, genetically encoded behavior of insects reshaped the fitness landscape for genes that affected development of pre-wings. After insect behavior had switched to a new state, it remained stable enough to control the direction of evolution of genes that encoded morphology. The behavioral switch itself took a long evolutionary time and involved irreversible genetic changes.

Because the interpretation system is more stable than the code, it cannot contribute to immediate fitness calculated locally for a considered portion of the code, but it may determine the rules of the evolutionary game. It can be viewed as an inference network consisting of interconnected configurable logical switches (Abel and Trevors 2006). This means that living systems use some sort of logic to interpret their encoded functional information and integrate it with signals coming from environmental sensors. I prefer to call it "inference" rather than "logic," because logic is usually associated with abstract axiomatic systems developed by humans, whereas living organisms have a diffuse network of inference rules which cannot be reduced to a small number of axioms. The idea that biological evolution follows internal inference rules is similar to the concept of directed evolution or nomogenesis (Berg 1969). Humans use logic to generate novel hypotheses that can then be tested in practice. A good logical system is supposed to generate a wide range of hypotheses with a high rate of passing subsequent testing steps. Similarly, natural interpretation systems that interpret the genome are supposed to produce diverse new biological functions with a high chance of getting established via genetic and habit selection. Thus, although the interpretation system makes no contribution to immediate utility, it may affect potential utility or adaptability (Conrad 1983).

Adaptability is a difficult notion to understand because there is no simple model like fitness landscape to explain it. It cannot be measured experimentally; instead it is assessed retrospectively on the basis of relative evolutionary success of different lineages. Each lineage evolves by developing tools for achieving life functions (e.g., 
locomotion or sense organs); however, some tools may appear more universal than others in terms of execution of additional functions. In the short term, it does not matter whether the tool is universal or not, but in the long run universal tools may accelerate evolution in various directions. Thus, universality of adaptations is the first component of adaptability. Universal status of adaptations may not appear at once; instead the structure or pathway may be reused later for a different purpose and its universality is increased gradually through this chain of events. For example, the initial function of a tail in vertebrates was to support swimming in fish. In terrestrial animals this function was lost; however, the tail appeared useful in many other ways (e.g., balancing, escaping predators, hanging on trees, signaling, etc.).

The second component of adaptability is making new functions compatible with already existing functions. Compatibility may include (1) protection from external effects of other functions, (2) minimizing the load on other functions, (3) support for other functions, and (4) combinatorial activation in various parts of the body by various signals. For example, high fecundity may solve the problem of increased mortality caused by a pathogen, but it increases the load on other functions (e.g., getting enough food). Developing an antimicrobial defense would be a better solution because it saves resources and is useful for fighting other infections. Another example of compatibility is functional redundancy between proteins. Many proteins can substitute for other members of the same family in various pathways and prevent system breakup. An example of combinatorial activation is the transformation of fly antennae into legs caused by a mutation in a single homeobox gene.

The third component of adaptability is accelerated pre-testing of new functions. For example, the success rate of genetic selection can be increased by habit selection via Baldwin's effect (Baldwin 1896). Habit selection substantially increases the variety of behavior patterns among individuals, and successful behaviors are preserved at least within the lifetime of an organism, and are occasionally copied by offspring or other members of the population. The logic of animal behavior, which is based on rewards and fear, is tuned well enough to select those actions that may eventually be beneficial for survival and reproduction. As these habits get selected, they can become encoded genetically and then organisms do not need to develop them by trial and error. In this case, the new function has already emerged and has been encoded in the brain; transferring it to the genome becomes a simple task, because all pathways for this function are ready and all that is left is to add corresponding changes to genes involved in these pathways. All fitness gradients are already set in the right direction; thus genetic selection becomes straightforward and fast. At this point there is no quantitative assessment of the importance of Baldwin's effect in evolution. But the fact that evolution of higher animals with a welldeveloped brain (e.g., mammals) was faster than in other lineages indicates that this effect may have been strong.

Evolution of interpretation systems is unpredictable, in contrast with the process of short-term genetic selection. However, one aspect of evolution of interpretation systems is predictable: it goes in the direction of higher adaptability. If some universal adaptation has emerged, then it does not disappear, as a rule, because lineages that carry this adaptation generate more diversity (species, genera, families), capture more resources, and eventually contribute more to future evolution. Thus, it 
is possible to consider long-term selection between different higher-level lineages (e.g. orders or classes), where success is measured not by survival and reproduction but by diversity, control of resources, and contribution to future evolution. Existing interpretation systems have survived in a long history of competition with other interpretation systems. Thus we should not be surprised by their ability to generate new functions efficiently and accelerate the process of genetic selection. It is not true that new functions generated by the interpretation system are always "adaptations for free" - parallel to the idea of "order for free" (Kauffman 1993) because interpretation systems passed the long test of lineage selection. Many evolutionary lineages went extinct because of their low adaptability, and that was the price paid for the high level of adaptability in those lineages that survived and prospered. But some new functions may indeed be "adaptations for free" because tools and pathways developed for specific needs may appear immediately useful for other purposes. Thus, it is possible that in some cases multifunctionality emerges spontaneously, without any prior selection.

Although adaptability is different from immediate utility, it can still be viewed as some kind of utility. It is useful because lineages with higher adaptability show greater evolutionary expansion. This process fits into an expanded version of Darwinism where selection is considered at the level of lineages rather than individual organisms (Eldredge 1985; Depew and Weber 1995). Thus we can distinguish multiple hierarchical levels of utility that differ in the time scale of their change (Fig. 1).

Habits encoded in the brain stand at the lowest level of the utility hierarchy because they serve to resolve immediate needs like finding resources or escaping from enemies. Short-term adaptations correspond to the medium level of the hierarchy and serve in optimization of functions needed for successful survival through the entire lifecycle. Long-term utility or adaptability belongs to a higher level and is needed for developing universal adaptations and novel functions. Biological functions developed at each level are encoded and interpreted by some inference system, but the inference system is optimized at a higher level of the hierarchy and at a longer time scale. In Fig. 1 I show only three main levels of

Fig. 1 Levels of utility and selection in the evolution of functional information

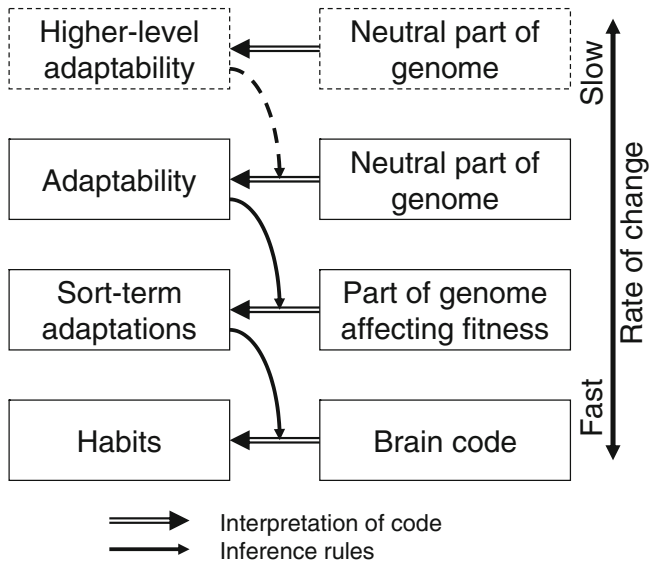


hierarchy; however, more levels may exist (e.g., shown by the dashed line). For example, epigenetically encoded lasting modifications can be placed at an intermediate level between behavioral and short-term genetic utility. There may be multiple levels of adaptability as well, each with a specific evolutionary time scale. We can hypothesize that each taxonomic level has a corresponding level of adaptability. Higher levels of adaptability control the most fundamental functions (e.g., hubs of molecular networks, body plans) characteristic of large taxa, whereas lower level of adaptability are of minor importance and are characteristic of small taxa.

\section{Communication System as a Carrier of Functional Information}

Because of the semantic closure, a successful interpretation of functional information (i.e., one that preserves a function) is possible only if communicating agents have similar/compatible interpretation systems. Thus, exchange of functional information is always limited to a set of compatible agents, which I propose to call communication system. The main idea is that functional information is not universal but circulates within a certain communication system and cannot be directly transferred to other communication systems. In particular, this idea challenges the common notion that human knowledge is universal and represents the only true description of reality (see discussion in the next section). Exchange of functional information increases the immediate utility of agents; however, the long-term evolution of a communication system is guided by inference rules of their shared interpretation system. If these inference rules support higher adaptability, then the communication system will prosper and produce divergent descendent communication systems.

It the simplest case, a communication system includes a single agent involved in self-communication with its own future states. Functional information is encoded and stored (i.e., in the brain or chromatin structure of cells) so that it can be reused in the future when needed. Self-communication is essential for self-identity of an agent. For example, self-identity of humans is based on the memory of our lives. When memory fails (e.g., as the result of trauma or psychiatric disorder), the identity is lost and the person/agent is no longer the same. Self-communication is essential for any agent because a system without self-communication is too simple to be called an agent. Genetic inheritance can be viewed as extended self-communication because the genome sequence is transferred to the daughter organism which is a part of the parent's self. A genome is a message used for long-term communication with all successive future generations. The purpose of communication is the same as for within-agent self-communication, which is to share functional information.

A more advanced type of communication involves horizontal transfer of messages between independent agents. In this case, the interpretation system encounters a code generated within a different interpretation system (in particular, within its encoding subsystem). The most common example of horizontal communication in living systems is sexual reproduction, when the maternal interpretation system (i.e., oocyte) encounters an unfamiliar paternal genetic sequence. Success of interpretation of the paternal genome copy depends on the similarity and compatibility of interpretation within all individuals of the population. Thus a population (or species) is a communication system with horizontal transfer of genetic messages. Organisms of 
different species do not belong to the same communication system because their interpretation systems are incompatible. Thus, most interspecies hybrids are nonviable or sterile as a result of misinterpretation of the paternal genome. Life on earth exists in the form of several million species, and each species is a carrier of unique functional information. So far, humans have learned only a small portion of this functional information. For example, we learned from fungi how to make antibiotics, and learned from plants how to produce narrowly targeted pyrethroid pesticides. If we want to learn more, then we need at least to preserve the biodiversity of natural communication systems.

Interestingly, small fragments of genomic sequence can be transferred between different bacterial species by viruses. In rare cases these fragments survive and acquire a new or modified function. The origin of transferred genes can be identified using sequence analysis (Koonin and Galperin 2003). Thus, exchange of functional information is possible even between different communication systems; however, these events are extremely rare compared with the frequency of information exchange within the same communication system.

Although interpretation systems of agents within the same communication system are compatible, they are not necessarily equal. For example, they may depend on the age (or stage) of development. Because communicating agents may differ in their interpretation ability, we need to distinguish simple interpretation, which is a straightforward processing of the code into action without any change of the interpretation system (no learning), and advanced interpretation, which involves multistep change of the interpretation system induced by the code. Simple interpretation is the most frequent kind of interpretation within the same communication system. Advanced communication is less frequent but it is necessary for growing (or recruiting) new members. For example, an egg contains the full genome but can interpret only a small portion of it. It can be compared to a child supplied with a library of encoded knowledge but who knows almost nothing except how to read. As the embryo develops, it learns how to extract functional information from the genome, starting with most simple functions. These functions allow the embryo to develop its morphology and cellular architecture, and individual daughter cells start specializing in their function. These specialized cells are capable of extracting additional functional information from the genome. This process takes a long time, but because it follows strict inference rules it is stable and eventually successful in extracting functional information from the genome.

Communication pathways can be very complex because some agents participate in multiple communication systems (e.g., self-communication, acoustic communication with neighbors, and genetic communication with progeny). In addition, most agents have hierarchical organization, and subagents may participate in exchange of functional information within a higher-level agent. Some agents (e.g., viruses) do not fully encode their interpretation systems. Thus, their communication system partially overlaps that of their host species.

\section{Human Functions and Knowledge: Reconciling Realism and Pragmatism}

Human functions are similar to biological functions in many respects. Our memory can be viewed as a code linked with an interpretation system in the brain which 
integrates the memory with incoming information from the environment and produces actions. Free will is a mutation in the mental code which is then processed through the interpretation system and emerges as a new (or modified) behavior. If we like the results of this behavior, the mutated mental code is preserved for future use. Thus, memory represents communication with our future states (i.e., self-communication) via selective storage of mental codes. For communication with other people we use another kind of code: spoken or written language. Translation from mental code to language and back is the subject of linguistics. It requires a complex thesaurus transferred between generations via education. A human communication system is a set of individuals who share similar/compatible interpretation systems. Normal exchange of messages does not require modification of interpretation system in recipients and therefore can be viewed as simple interpretation (i.e., it lacks any new meaning). However, human communication systems need to recruit new members to remain stable or to expand, and this requires advanced interpretation (e.g., education).

Let us consider factors of human evolution and compare them with factors of biological evolution discussed above. The major novel factor of human evolution is immortalization of a large portion of the mental code which survives in many generations via a potentially indefinite transmission. Elements of this code, called "memes," are copied to the mental state of message recipients, and recipients then communicate these memes to other people (Dawkins 1989). Memes are often transferred to a written text, which increases their stability and proliferation. Utility is definitely a very important factor in human evolution. Ancient humans acquired memes that encoded how to make fire, domesticate animals, and grow crops. This knowledge positively affected food production and human population growth. Recent technological innovations have brought even greater productivity and improved quality of human life. Interestingly, utility criterion has shifted in human evolution: it is now linked more with the quality of life than with survival and reproduction (Dawkins 1989). This happened because contemporary human evolution is driven mostly by habit selection rather than by genetic selection. Habits are communicated via language (memes), and this process is entirely independent from differential survival and reproduction. There is still some role of sexual selection, but it is much slower than selection of technology and lifestyle.

One of the greatest achievements of the human mind was the development of logic, which can be viewed as an advanced interpretation system with structured inference rules. The basic idea is to accumulate a large set of true statements (knowledgebase) which can then be combined in different ways using logical operators to produce new true statements. According to the philosophy of realism (and logical positivism), a statement is true if it matches reality. The statement "snow is white" is true if snow is indeed white (correspondence theory of Wittgenstein and Tarski). Realism denies contradictions within reality; hence the knowledgebase should be all logically coherent. The main problem of realism is that it has no tolerance for contradictions. If a contradiction is found, then the entire knowledgebase should be discarded because the source of the problem is not clear. Realism overemphasizes the role of logic in the growth of knowledge. For example, physicists often interpret their models as Laws of Nature, as if these laws were embedded in reality. But a closer look reveals that there are always competing models that match reality equally well. Some of these models work better in one area; others are more useful in another area. 
An alternative approach to realism is the philosophy of pragmatism, founded by Peirce (1955), James (1954), and Dewey (1998). According to pragmatism, usefulness is the most reliable criterion of truth. Correspondence theory of truth may work well within a specific communication system where all notions are fixed and coherent among people. But it fails if applied to communication across cultures and languages because notions and even social functions do not match. Definitions don't help to resolve the problem because the words that are used in definitions also do not match exactly between cultures and languages. But pragmatism can be readily applied to communication across cultures. The message is understood correctly if the recipient can reproduce the transmitted function to a level that is useful. For example, we can barely understand a few words in a foreign language but are still able to understand directions to the railway station. Pragmatism tolerates limited contradictions within an interpretation system. Thus there is no reason to discard a knowledgebase with internal logical problems because most functional information can still be transferred successfully. Of course, contradictions decrease the utility of a knowledgebase and it is better to reduce them to a minimum. Fuzzy logic may be useful for measuring the integrity of a knowledgebase and minimizing contradictions (Zadeh 1989).

The main weakness of pragmatism stems from an underestimation of the role of logic. Because utility is considered the main criterion of truth, there is no real need for logic. In this respect, pragmatism is similar to neo-Darwinism, which assumes a dominating role of genetic selection (i.e., utility) in biological evolution. Because realism and pragmatism overemphasize different factors of evolution of functional information (i.e., inference and utility), it seems natural to integrate both into one concept that can be called evolutionary pragmatism (or pragmatic constructivism). This synthesis rejects the main assumption of realism that logic is embedded in reality. Instead, logic is constructed by humans as a tool for efficient derivation of new knowledge. Logic is a part of the knowledgebase and evolves together with it. The role of utility in the evolution of human knowledge is highest in the areas of practical importance (e.g., development of new technology).

Similarly to biological interpretation systems, the human mental interpretation system and logic evolved towards higher adaptability (e.g., a higher rate of innovation, multifunctionality, integrity, and reliability). Elements of thesaurus and/ or logic were replaced or modified to increase the adaptability. Axiomatic systems offer a powerful method for avoiding logical contradictions; thus this method was readily accepted in mathematics and some areas of physics. However, any statement derived via logic is not automatically true by definition. It should always be considered a hypothesis with at least some element of doubt. This does not mean that we have to test every hypothesis generated by logic. In many cases we do not test them because the risk of being wrong is too small or the cost of testing too high. This version of evolutionary pragmatism acknowledges the crucial role of inference and logic in the evolution of human knowledge, but at the same time it retains the idea of the importance of utility as a reality check component.

Acknowledgements I thank Alexey Voinov (University of Vermont, Burlington, VT) and Olga Brazhnik (National Institute of Health, Bethesda, MD) for discussion of the manuscript. This work was supported by the Intramural Research Program of the National Institute on Aging, NIH. 


\section{References}

Abel, D. L., \& Trevors, J. T. (2006). Self-organization vs. self-ordering events in life-origin models. Physics of Life Reviews, 3, 211-228.

Ackoff, R. L., \& Emery, F. E. (1972). On purposeful systems. Chicago, New York: Aldine-Atherton.

Ahituv, N., Zhu, Y., Visel, A., Holt, A., Afzal, V., Pennacchio, L. A., et al. (2007). Deletion of ultraconserved elements Deletion of ultraconserved elements yields viable mice. PLoS Biology, 5(9), e234.

Berg, L. S. (1969). Nomogenesis. Cambridge: M.I.T.

Baldwin, M. J. (1896). A new factor in evolution. American Naturalist, 30, 441-451.

Bouché, N., \& Bouchez, D. (2001). Arabidopsis gene knockout: phenotypes wanted. Current Opinion in Plant Biology, 4(2), 111-117.

Conrad, M. (1983). Adaptability. The significance of variability from molecule to ecosystem. New York: Plenum.

Dawkins, R. (1989). The Selfish Gone (2nd ed.). Oxford: Oxford University Press.

Depew, D. J., \& Weber, B. H. (1995). Darwinism evolving. Systems dynamics and the genealogy of natural selection. Cambridge: M.I.T.

Dewey, J. (1998). The development of American pragmatism. In L. A. Hickman, \& T. M. Alexander (Eds.), The essential Dewey. Vol. 1. Bloomengton: Indiana Univ. Press.

Eldredge, N. (1985). Unified synthesis: Biological hierarchies and modern evolutionary thought. Oxford, New York: Oxford University Press.

Fisher, R. A. (1930). The genetical theory of natural selection. Oxford: Clarendon.

Hoffmeyer, J. (1996). Signs of meaning in the Universe. Bloomington: Indiana University Press.

James, W. (1954). Essays in pragmatism. Edited by A. Castell. New York: Hafner Pub. Co.

Kauffman, S. A. (1993). The origins of order: Self-organization and selection in evolution. New York: Oxford Univ. Press.

Kauffman, S. A. (2000). Investigations. Oxford, New York: Oxford Univ. Press.

Koonin, E. V., \& Galperin, M. Y. (2003). Sequence - evolution - function. Computational approaches in comparative genomics. Boston, London: Kluwer Acad. Publ.

Pattee, H. H. (1982). Cell psychology: an evolutionary approach to the symbol-matter problem. Cognition and Brain Theory, 5, 325-341.

Peirce, C. S. (1955). Philosophical writings of Peirce. Edited by J. Buchler. New York: Dover Publications.

Rosen, R. (1985). Anticipatory systems. Oxford, New York, Paris: Pergamon.

Rosen, R. (1991). Life itself. New York: Columbia University Press.

Sharov, A. A. (1991). Self-reproducing systems: structure, niche relations and evolution. BioSystems, 25, $237-249$.

Sharov, A. A. (1992). Biosemiotics: A functional-evolutionary approach to the analysis of the sense of information. In T. A. Sebeok, \& J. Umiker-Sebeok (Eds.), Biosemiotics: The Semiotic Web 1991 (pp. 345-373). Berlin: Mouton de Gruyter.

von Uexküll, J. (1982). The Theory of Meaning. Semiotica, 42(1), 25-82.

Zadeh, L. A. (1989). Knowledge representation in fuzzy logic. IEEE Transactions on Knowledge and Data Engineering, 1, 89-100. 